\title{
AVALIAÇÃO DE PRODUTOS OBTIDOS PELA DESIDRATAÇÃO OSMÓTICA DE BANANA SEGUIDA DE SECAGEM
}

\author{
PAULO HENRIQUE MACHADO DE SOUSA * \\ GERALDO ARRAES MAIA ** \\ MEN DE SÁ MOREIRA DE SOUZA FILHO *** \\ RAIMUNDO WILANE DE FIGUEIREDO ** \\ RENATA TIEKO NASSU *** \\ MARIA DE FÁTIMA BORGES ***
}

\begin{abstract}
Foram obtidos dois produtos de banana por desidratação osmótica, um sob pressão atmosférica e outro sob vácuo, com complemento de secagem em estufa. A estabilidade dos produtos foi estudada, durante 120 dias, mediante análises físico-químicas, microbiológicas e sensoriais. Nesse período, os produtos ficaram armazenados em temperatura ambiente $\left(23,4-34,1^{\circ} \mathrm{C}\right)$ e umidade relativa entre $33-81 \%$. Concluiu-se que os produtos mantiveram suas características químicas, físico-químicas e sensoriais, além de estabilidade microbiológica (pouca variação durante o período de armazenamento). Somente a cor dos produtos apresentou variação expressiva. Sugere-se o tratamento utilizando osmose com vácuo seguido de secagem para produção de banana passa.
\end{abstract}

PALAVRAS-CHAVE: BANANA-ANÁLISES FÍSICO-QUÍMICAS; DESIDRATAÇÃO OSMÓTICA; BANANA-DESIDRATAÇÃO.

\section{INTRODUÇÃO}

A banana (Musa spp.) é uma das frutas mais consumidas no mundo, sendo produzida na maioria dos países tropicais. Em 2000, a produção mundial chegou a 64 milhões de toneladas, destacando-se a Índia como o principal país produtor. O Brasil, maior consumidor mundial, ocupa o terceiro lugar (depois do Equador, segundo produtor mundial) com 10\% da produção total (FAO, 2001).

* Bolsista de Extensão Tecnológica, Fundação Cearense de Apoio ao Desenvolvimento Científico e Tecnológico (FUNCAP), Universidade Federal do Ceará, Fortaleza, CE (e-mail:phmachado@uol.com.br).

** Professores, Universidade Federal do Ceará, Fortaleza, CE (e-mail: frutos@ufc.br).

*** Pesquisadores, Embrapa Agroindústria Tropical, Fortaleza, CE (e-mail: sa@cnpat.embrapa.br). 
Sob o ponto de vista biológico, a banana apresenta uma das maiores perdas na produção, devido à decomposição pós-colheita. Sendo extremamente perecível não permite o uso do frio para o armazenamento. Tal fato sugere a industrialização como alternativa para melhorar o aproveitamento da produção. Dentre os processos industriais de aproveitamento da fruta, a produção de banana passa merece destaque devido ao baixo investimento inicial e perspectiva de lucratividade compatível com o investimento. Além disso, o mercado permite a absorção de grande volume do produto em relação à oferta real (SILVA, 1995).

O método de desidratação osmótica de alimentos consiste na remoção parcial de água pela pressão osmótica, e vem sendo utilizado para conservação de frutas, verduras, carnes e pescados. O alimento é colocado em contato com solução hipertônica de solutos, diminuindo a atividade de água. Aumenta-se sua estabilidade em combinação com outros fatores como controle de $\mathrm{pH}$, adição de antimicrobianos, etc. (POKHARKAR et al., 1997). Além do fluxo de água do alimento para a solução, existe o fluxo do soluto para o alimento. Porém, o interesse no processo reside no fato de que o fluxo de água é maior que o fluxo de soluto (MIZRAHI et al., 2001).

As principais vantagens do processo de desidratação osmótica sobre os processos tradicionais de secagem são a inibição do escurecimento enzimático, com retenção da cor natural sem a utilização de sulfitos, maior retenção de componentes voláteis durante a secagem subseqüente e baixo consumo de energia (MAEDA e LORETO, 1998; POKHARKAR et al., 1997).

Quando o trabalho é feito com pressões inferiores à atmosférica obtémse desidratação osmótica a vácuo. O estudo da sua aplicação em frutas e hortaliças revelou características vantajosas para o uso industrial. A desidratação osmótica a vácuo apresenta-se como avanço no processo de desidratação osmótica. Contribui para acelerar a perda de água em comparação com o tratamento em pressão normal, torna o processo mais rápido e possibilita a obtenção de frutos desidratados de boa qualidade (SHI e FITO, 1993; SHI et al., 1995).

A desidratação osmótica tem sido bastante utilizada como pré-tratamento para a secagem convectiva de frutas. Apesar da secagem oferecer longa vida de prateleira pode-se observar decréscimo na qualidade do produto final. A desidratação osmótica minimiza os efeitos adversos que geralmente aparecem com a utilização dos métodos convencionais, como dureza 
excessiva, degradação da cor, aroma e sabor, além de dificuldade na reidratação. Também estão relacionados com a melhoria de muitas propriedades nutricionais e funcionais dos produtos quando comparados com outros processos de desidratação direta (TORREGGIANI, 1993).

Neste trabalho pretendeu-se obter produtos de banana por desidratação osmótica (com e sem a utilização de vácuo) como pré-tratamento para a secagem em estufa e avaliar sua estabilidade pelo período de 120 dias.

\section{MATERIAL E MÉTODOS}

Bananas da variedade prata (Musa sapientum $L$.), procedentes da Central de Abastecimento S/A do Ceará (CEASA-CE), foram recebidas em planta piloto e selecionadas de acordo com seus atributos de qualidade. Tais atributos incluíam a cor (amarelas sem pontos pretos), a uniformidade, o grau de maturação (estágio 3/4 gorda) e a isenção de defeitos. Em seguida, os frutos foram lavados por imersão em água clorada (50 ppm de cloro ativo/15 min). As bananas foram descascadas manualmente, raspadas para retirada do mesocarpo, e cortadas as pontas para se evitar adstringência devido à grande concentração de taninos nas extremidades da fruta. Em seguida, os frutos foram submetidos a branqueamento com vapor saturado $\mathrm{a} 100^{\circ} \mathrm{C}$ por 2 minutos e à desidratação osmótica. Usouse xarope de sacarose a $65^{\circ}$ Brix na proporção fruto:xarope $1: 2$ a $65^{\circ} \mathrm{C}$ sob duas condições: pressão atmosférica por 3 horas de imersão no xarope (T1) e imersão sob vácuo $(660 \mathrm{mmHg})$ por 1 hora e $15 \mathrm{~min}$ (T2). Ajustou-se o pH do xarope pela adição de ácido cítrico até 3 e usou-se benzoato $(0,1 \%)$ como conservante.

Após a osmose, os frutos foram retirados do xarope de sacarose e colocados em estufa com circulação de ar forçada, marca MARCONI, modelo MA 035 , a $65^{\circ} \mathrm{C} \pm 5^{\circ} \mathrm{C}$ por $16 \mathrm{~h}$ (T1) e $11 \mathrm{~h}$ (T2). Os produtos obtidos foram embalados em recipientes retangulares de polietileno tereftalatado (PET) transparentes, com tampa (NEOFORM ${ }^{\odot} \mathrm{N}-94$ ) e capacidade para $200 \mathrm{~g}$ do produto. Os recipientes foram armazenados em temperatura ambiente $\left(23-24^{\circ} \mathrm{C}\right)$ e umidade relativa de $33-81 \%$ por 120 dias.

A avaliação da estabilidade foi realizada mediante análises físico-químicas. $A$ atividade de água foi medida em aparelho Aqualab (CX-2 da Decagon), o pH em pHmetro digital e o conteúdo de umidade pela evaporação direta a $70^{\circ} \mathrm{C}$ (AOAC, 1992). Determinou-se a acidez total titulável mediante titulação com hidróxido de sódio (expressa em \% de ácido cítrico) e o 
teor de sólidos solúveis totais em refratômetro de bancada modelo ABBÉ (expressa em ${ }^{\circ}$ Brix) conforme as Normas Analíticas do Instituto Adolfo Lutz (1985). Determinou-se a cor em colorímetro Minolta (Color Meter CR300), sendo expressa como valor $L^{*}$.

As análises microbiológicas de contagem de microrganismos aeróbios mesófilos, bolores e leveduras, coliformes a $35^{\circ} \mathrm{C} / \mathrm{g}$ e Coliformes a $45^{\circ} \mathrm{C} / \mathrm{g}$, Escherichia coli e Salmonella sp. foram efetuadas de acordo com a APHA (2001). A avaliação sensorial envolveu 30 julgadores não-treinados e teste de aceitação. Utilizou-se escala hedônica de 9 pontos, ancorada nos extremos em "gostei muitíssimo" (9) e "desgostei muitíssimo" (1) conforme MONTEIRO (1984). A análise de variância e o teste de Tukey foram utilizados para determinar diferença estatística significativa $(p \leq 0,05)$ entre as médias (SAS, 1996).

\section{RESULTADOS E DISCUSSÃO}

As características físico-químicas dos produtos pré-tratados à pressão atmosférica (T1) e sob vácuo (T2) não diferiram estatisticamente (Teste de Tukey, ao nível de $5 \%$ de significância), exceto para o teor de sólidos solúveis totais ( ${ }^{\circ}$ Brix) e o valor $L^{*}$ (Tabela 1 ). Tal fato evidencia maior incorporação de sólidos solúveis no tratamento 2 , facilitado pelo vácuo.

\section{TABELA 1 - CARACTERÍSTICAS FÍSICO-QUÍMICAS DOS PRODUTOS DE BANANA PRÉ-TRATADA POR DESIDRATAÇÃO OSMÓTICA À PRESSÃO ATMOSFÉRICA E SOB VÁCUO, SEGUIDA DE SECAGEM EM ESTUFA NO TEMPO ZERO}

\begin{tabular}{lcc}
\hline \multirow{2}{*}{ Determina I es } & \multicolumn{2}{c}{ Tempo Zero } \\
\cline { 2 - 3 } $\mathrm{pH}$ & $\mathbf{T}$ & T2 \\
\hline S lidos solœreis $\left({ }^{\circ}\right.$ Brix $)$ & $71,3^{\mathrm{a}}$ & $4,05 \mathrm{a}$ \\
Acidez $(\%$ de Aeido) & $0,81^{\mathrm{a}}$ & $68,5 \mathrm{~b}$ \\
Umidade $(\% \mathrm{p} / \mathrm{p})$ & $17,8^{\mathrm{a}}$ & $0,71 \mathrm{a}$ \\
Aa & $0,721^{\mathrm{a}}$ & $18,7 \mathrm{a}$ \\
Cor (valor $\left.\mathrm{L}^{*}\right)$ & $52,80 \mathrm{a}$ & $0,736 \mathrm{a}$ \\
\hline
\end{tabular}

Amostras seguidas pelas mesmas letras na mesma linha não diferem $(p>0,05)$ pelo teste de Tukey.

$\mathrm{T} 1$ = Produto tratado com osmose à pressão atmosférica seguida de secagem em estufa.

T2 = Produto tratado com osmose sob vácuo seguido de secagem em estufa.

$\mathrm{Aa}=$ Atividade de água . 
O maior valor de $L^{*}$ de $T 2$ indica maior escurecimento durante o processo sob vácuo, o qual ocorre pela maior quantidade de sólidos solúveis presentes. No último mês de armazenamento (120 dias), a comparação entre os produtos (T1 e T2) mostrou que os resultados diferiram estatisticamente ao nível de 5\% (Tabela 2) apenas para os valores de acidez titulável e cor (valor $\left.L^{*}\right)$.

\section{TABELA 2 - CARACTERÍSTICAS FÍSICO-QUÍMICAS DOS PRODUTOS DE BANANA PRÉ-TRATADA POR DESIDRATAÇÃO OSMÓTICA À PRESSÃO ATMOSFÉRICA E SOB VÁCUO, SEGUIDA DE SECAGEM EM ESTUFA NO TEMPO DE 120 DIAS}

\begin{tabular}{lcc}
\hline \multirow{2}{*}{ Determina I es } & \multicolumn{2}{c}{ Tempo 120 dias } \\
\cline { 2 - 3 } & T1 & T2 \\
\hline $\mathrm{pH}$ & $4,25 \mathrm{a}$ & $4,27 \mathrm{a}$ \\
S lidos soloreis ( ${ }^{\circ}$ Brix $)$ & $73,5 \mathrm{a}$ & $74,5 \mathrm{a}$ \\
Acidez (\% de Aeido c trico) & $1,18 \mathrm{a}$ & $1,01 \mathrm{~b}$ \\
Umidade (\%p/p) & $16,8 \mathrm{a}$ & $16,4 \mathrm{a}$ \\
Aa & $0,695 \mathrm{a}$ & $0,670 \mathrm{a}$ \\
Cor (valor $\left.\mathrm{L}^{*}\right)$ & $33,44 \mathrm{a}$ & $29,39 \mathrm{~b}$
\end{tabular}

Amostras seguidas pelas mesmas letras na mesma linha não diferem $(p>0,05)$ pelo teste de Tukey.

$\mathrm{T} 1=$ Produto tratado com osmose à pressão atmosférica seguida de secagem em estufa.

T2 = Produto tratado com osmose sob vácuo seguido de secagem em estufa.

$\mathrm{Aa}=$ Atividade de água .

Somente os valores obtidos nas determinações de sólidos solúveis e de atividade de água para o produto do $\mathrm{T} 1$ não apresentaram diferença significativa $(p>0,05)$ no decorrer do tempo de armazenagem. O restante das determinações ( $\mathrm{pH}$, acidez total titulável, umidade e cor) do produto T1 e todas as determinações do produto T2 apresentaram diferença significativa $(p \leq 0,05)$ durante o armazenamento (Figuras 1 a 6$)$.

O pequeno aumento no valor do $\mathrm{pH}(4,03-4,25$ para $\mathrm{T} 1$ e 4,05-4,27 para T2) durante o tempo de armazenamento pode ser atribuído à dissociação do ácido cítrico (Figura 1). 
FIGURA 1 - VARIAÇÃO DO pH EM FUNÇÃO DO TEMPO DE ARMAZENAMENTO



FIGURA 2 - VARIAÇÃO DOS SÓLIDOS SOLÚVEIS ( BRIX) EM FUNÇÃO DO TEMPO DE ARMAZENAMENTO

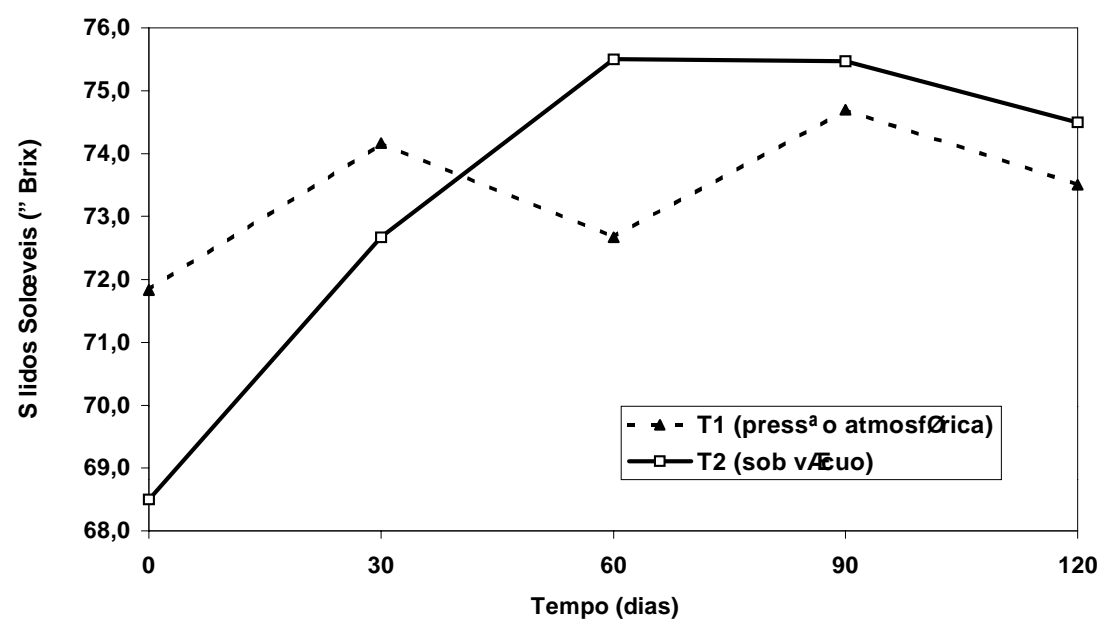


FIGURA 3 - VARIAÇÃO DA ACIDEZ EM FUNÇÃO DO TEMPO DE ARMAZENAMENTO

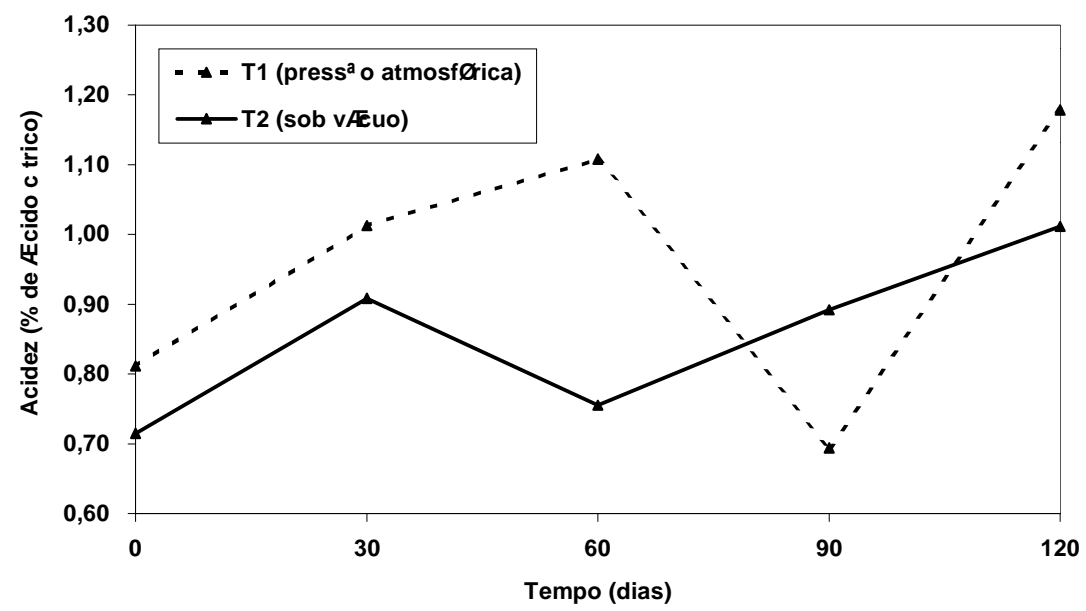

FIGURA 4 - VARIAÇÃO DA UMIDADE EM FUNÇÃO DO TEMPO DE ARMAZENAMENTO

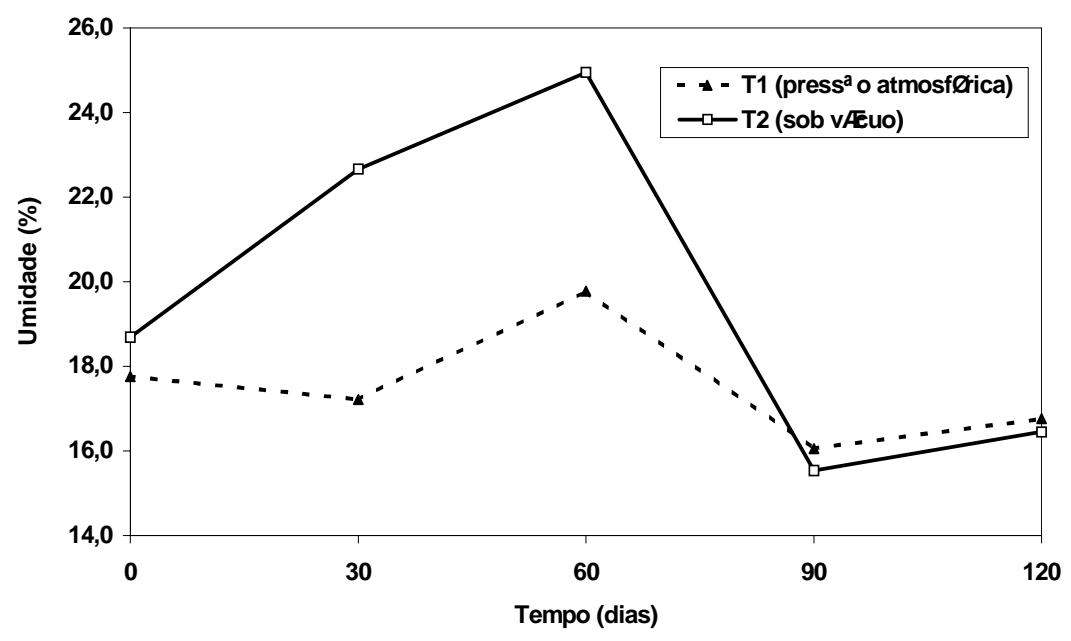


FIGURA 5 - VARIAÇÃO DA ATIVIDADE DE ÁGUA EM FUNÇÃO DO TEMPO DE ARMAZENAMENTO

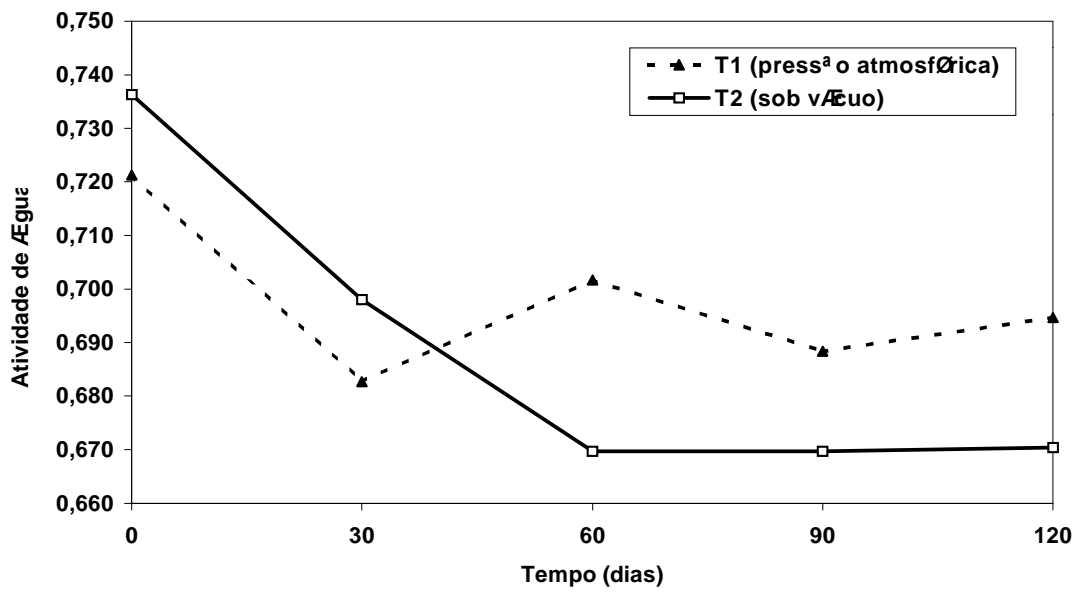

FIGURA 6 - VARIAÇÃO DA COR (VALOR L*) EM FUNÇÃO DO TEMPO DE ARMAZENAMENTO

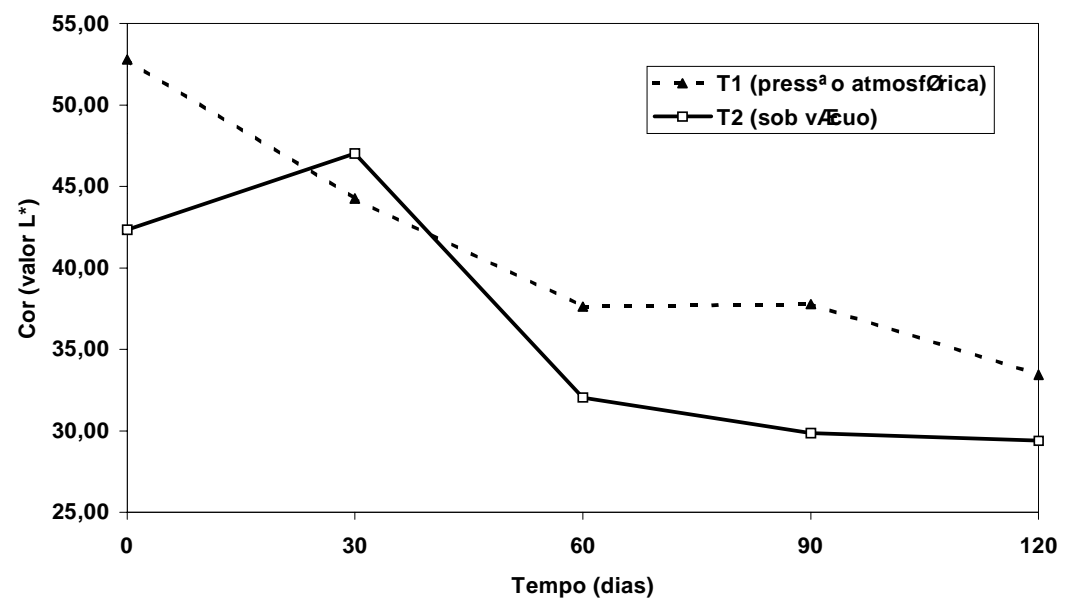


Pode-se explicar a variação da acidez (0,68-1,18 para T1 e 0,71-1,01 para T2) pela degradação do ácido cítrico ou pela variação do conteúdo de umidade ao longo do armazenamento, que influencia o cálculo da acidez total titulável (Figura 3).

A variação da umidade (17,8-16,8 para T1 e 18,7-16,4 para T2) e da atividade de água (0,695-0,721 para T1 e 0,736-0,670 para T2) no decorrer do tempo de armazenamento pode ser atribuída à relativa permeabilidade das embalagens PET utilizadas. Essas embalagens facilitam as trocas com o ambiente, cujo comportamento varia conforme a umidade relativa e a temperatura do local de armazenamento (Figuras 4 e 5).

Os valores de $L^{*}$ variaram entre 33,44 e 52,79 para T1 e entre 29,39 e 47,01 para T2 (Figura 6), indicando maior tendência ao escurecimento (redução do valor $L^{*}$ ) quanto maior o tempo de armazenamento. Os valores de $L^{*}$ variam de 0 (preto) a 100 (branco) devido ao escurecimento do produto por meio de rotas enzimáticas e não-enzimáticas. Tal fato pode ser justificado pela grande quantidade de açúcares presente nos produtos e pela ausência de aditivos para preservação da cor.

Durante todo o período de armazenamento, os produtos apresentaram contagem de microrganismos aeróbios mesófilos inferiores a 10 UFC/g, bem como bolores e leveduras inferiores a $10 \mathrm{UFC} / \mathrm{g}$. As determinações de Coliformes a $35^{\circ} \mathrm{C}$, Coliformes a $45^{\circ} \mathrm{C}$ e E. coli resultaram em valores inferiores a $3 \mathrm{NMP} / \mathrm{g}$. A presença de Salmonella sp. não foi detectada nas amostras avaliadas. As amostras atenderam à legislação brasileira em vigor (BRASIL, 2001), que estabelece os seguintes padrões microbiológicos para frutas desidratadas com adição de açúcares: máximo de $10^{2} \mathrm{NMP} / \mathrm{g}$ para Coliformes a $45^{\circ} \mathrm{C}$ e ausência de Salmonella sp. em $25 \mathrm{~g}$ do produto.

Os resultados encontrados após o processamento e durante o armazenamento podem ser atribuídos ao valor baixo de $\mathrm{pH}$, presença de aditivos como benzoato de sódio $(0,1 \%)$ e ácido cítrico, além de baixa atividade de água $(\mathrm{Aa})$. Tais características dos produtos são desfavoráveis para o desenvolvimento da maioria dos microrganismos.

AZEREZO e JARDINE (2000) encontraram resultados semelhantes em estudo com abacaxis desidratados osmoticamente. Os valores das contagens de bolores e leveduras foram inferiores a $10 \mathrm{UFC} / \mathrm{g}$ ao longo de 60 dias de armazenamento a $30^{\circ} \mathrm{C}$.

A Figura 7 apresenta o somatório das notas 6/7/8/9 da aceitação global 
das amostras de desidratados de banana após processamento. Foi observada maior freqüência de notas acima de 6 para os dois experimentos, sendo mais apreciado o produto correspondente ao experimento T2 (sob vácuo). Os comentários dos provadores indicaram que o produto do T2 mostrou "melhor aparência", "sabor caramelizado" e umidade mais elevada que o do T1.

\section{FIGURA 7 - REPRESENTAÇÃO GRÁFICA DO SOMATÓRIO DAS FREQÜÊNCIAS DE NOTAS 6/7/8/9 EM FUNÇÃO DO TEMPO DE ARMAZENAMENTO}

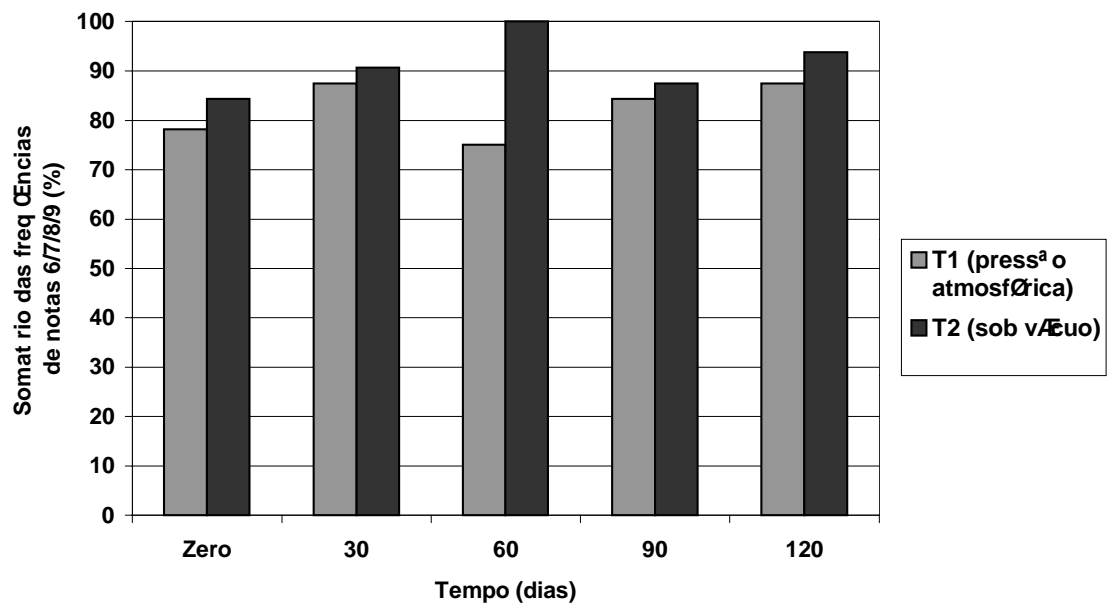

Segundo LABUZA e SCHIMDL (1988) considera-se o final da vida útil de determinado produto quando ocorre queda de 1,5 pontos entre os resultados da escala hedônica, o que não aconteceu em nenhum dos experimentos. A variação da avaliação global foi de 6,5-6,7 para T1 e 7,07,6 para T2 durante o tempo de armazenamento, evidenciando que as amostras foram consideradas aceitáveis pelos provadores durante o período estudado (120 dias).

\section{CONCLUSÃO}

Os produtos desidratados obtidos a partir da banana mantiveram suas características físico-químicas com pouca variação durante 120 dias de 
armazenamento em temperatura ambiente. A variação mais expressiva foi observada quanto a cor dos produtos.

A estabilidade microbiológica indicou que os produtos de banana desidratados por pré-tratamento osmótico seguido de secagem em estufa foram processados em condições higiênico-sanitárias adequadas. A metodologia empregada mostrou-se efetiva na prevenção do desenvolvimento microbiano pelo período de 120 dias.

Os resultados da avaliação sensorial das bananas desidratadas (durante 120 dias de armazenamento) indicaram boa aceitação, com pouca diferença entre os produtos. A banana tratada previamente com osmose a vácuo apresentou maior aceitação pelos julgadores.

Sugere-se a desidratação osmótica sob vácuo, seguida de secagem, para obtenção de banana passa por exigir menor tempo de imersão dos frutos no xarope e menor tempo de secagem, além de melhor aceitação dos produtos.

\section{Abstract}

\section{EVALUATION OF PRODUCTS OBTAINED BY THE BANANA'S OSMOTIC} DEHYDRATION FOLLOWED OF DRYING

It was obtained two bananas products through osmotic dehydration, one under atmospheric pressure and other under vacuum, with drying complement in stove. The storage stability of these products was studied, during 120 days, through physicalchemical, microbiological and sensorial analysis. In this period, the products were stored at room temperature $\left(23,4-34,1^{\circ} \mathrm{C}\right)$ and relative humidity between $33-81 \%$. It was concluded that the obtained products maintained their chemical, physico-chemical and sensory characteristics, beyond microbiological stability (small variations during the storage period). Only the color of the produtcs presented more expressive variations. Therefore it is suggested the treatment using osmosis with vacuum followed by oven drying for the production of banana fig.

KEY-WORDS: BANANAS-PHYSICO-CHEMICAL ANALYSIS; OSMOTIC DEHYDRATION; BANANA-DEHYDRATION.

\section{REFERÊNCIAS}

1 AOAC. Association of Official Analytical Chemists. Official methods of analysis of the AOAC. $12^{\text {th }}$ ed. Washington, $1992.1115 \mathrm{p}$.

2 APHA. American Public Health Association. Compendium of methods for the microbiological examination of foods. Washington, DC, 2001. $676 \mathrm{p}$.

3 AZEREZO, H.M.C.; JARDINE, J.G. Desidratação osmótica de abacaxi aplicada à 
tecnologia de métodos combinados. Ciência e Tecnologia de Alimentos, v. 20, n. 1 , p. $74-82,2000$.

4 BRASIL. Ministério da Saúde. Agência Nacional de Vigilância Sanitária (ANVISA). Resolução RDC no 12, de 02 de janeiro de 2001. Dispõe sobre os princípios gerais para o estabelecimento de critérios e padrões microbiológicos para alimentos. Disponível em: <http://www.vigilanciasanitaria.gov.br/anvisa.html>. Acesso em 20 ago. 2001.

5 FAO. FAOSTAT: database result: banco de dados. Disponível em: <http:// www.fao.gov.br>. Acesso em 20 ago. 2001.

6 INSTITUTO ADOLFO LUTZ. Normas analíticas do Instituto Adolfo Lutz: métodos químicos e físicos para análises de alimentos. 3.ed. São Paulo, 1985. v. 1.

7 LABUZA, T.P. e SCHIMDL, M.K. Use of sensory data in the shelf life testing of foods: principles and graphical methods for evaluation. Cereals Foods World, St. Paul, v. 33, n. 2, p. 193-206, Feb., 1988.

8 MAEDA, M.; LORETO, R.L. Desidratação osmótica de bananas. Semina: Ci. Agr., Londrina, v. 19, n.1, p. 60-67, mar. 1998.

9 MIZRAHI, S.; EICHLER, S.; RAMON, O. Osmotic dehydration phenomena in gel systems. Journal of Food Engineering, n. 49, p. 87-96, 2001.

10 MONTEIRO, C.B.L. Técnicas de avaliação sensorial. 2.ed. Curitiba: UFPR/CEPPA, 1984. $101 \mathrm{p}$.

11 POKHARKAR, S.M.; PRASAD, S.; DAS, H. A model for osmotica concentration of bananas slices. Journal Food Science and Technology, v. 34, n. 3, p. 230-232, 1997.

12 SAS Institute. Statistics analysis systems (SAS). Cary, NC, 1996.

13 SHI, X.Q.; FITO, P.; CHIRALT, A. Influence of vacuum treatment on mass transfer during osmotic dehydration of fruits. Food Research International, v. 28, n. 5, p. 445-54, 1995.

14 SHI, X.Q.; FITO, P. Vacuum osmotic dehydration of fruits. Drying Technology, v. 11, n. 6, p. 1429-1442, 1993.

15 SILVA, C.A.B. (Coord.). Produção de banana passa. Brasília: Ministério da Agricultura, do Abastecimento e da Reforma Agrária, Secretaria do Desenvolvimento Rural, 1995. 32 p. (Série Perfis Agroindustriais, v. 5).

16 TORREGGIANI, D. Osmotic dehydration in fruit and vegetable processing. Food Research International, v. 26, p. 59-68, 1993. 